\title{
Repoblación y sociedad en el reino de Mallorca (1230-1343)
}

\author{
Alvaro Santamaria *
}

\section{PECULIARIDADES DE LA CONQUISTA}

1. La repoblación cristiana del reino de Mallorca se enmarca en el proceso de la reconquista hispánica peninsular, dada la función del archipiélago de las Baleares, reiterada en las fuentes, como antemural peninsular y avanzada de España por lo menos desde la época romana; y, paralelamente, se inscribe en el proceso de recuperación cristiana de las vías marítimas mediterráneas dada la situación del espacio balear, de Mallorca concretamente, definida por los nevantes medievales como cap de creus, en el sentido de encrucijada marítima Norte-Sur y Este-Oeste.

La recuperación la promueve la Corona de Aragón al amparo de derechos históricos implícitamente reconocidos en el Tratado de Cazorla de 1179 sobre el reparto de áreas de influencia entre la Corona de Castilla y la Corona de Aragón. Y el operativo se programa en 1228 y se realiza en 1229 aprovechando circunstancias favorables propiciadas sobre todo por el vacío de mando que genera en la España musulmana y en el Mediterráneo occidental el progresivo deterioro del imperio almohade a partir de las Navas de Tolosa.

La realización del operativo Mallorca/1229, aunque incardinada en el marco de la reconquista hispánica peninsular presenta peculiaridades que consideraremos seguidamente.

\footnotetext{
* Universidad de las Islas Baleares.
} 


\section{Planificación del operativo como empresa asociada}

2. No es hecho singular que la Corona pacte una operación de conquista con los poderes que tienen recursos para acometerla. Lo singular es que el pacto se concrete en acuerdo multitateral abierto en el que los participantes, desde una mentalidad inversionista, se obligan a aportar recursos para participar en los beneficios que se obtengan en proporción a los recursos aportados; beneficios, en su caso, compensatorios de los capitales invertidos y de los riesgos asumidos; a repartir los beneficios con equidad, concretamente a la par, en reparto entre iguales inter pares.

En el contrato ya se nomina -con nueve meses de antelación al arranque del operativo-, la Comisión de repartidores constituida por magnates eclesiásticos y seglares catalanes, roselloneses y bearneses; luego, se incorporan a dicha comisión dos barones aragoneses que de esta manera quedó integrada por ocho miembros.

En el primer convenio (Barcelona, 23-XII-1228) la operación se presenta como abierta sólo a súbditos de la Corona; pero al producirse la empresa como cruzada, amparada de una bula de cruzada y en el marco de la confrontación Islam-Cristiandad, al concluirse el convenio definitivo (Tarragona, 28-VIII-1229) a la mención inicial omnes homines de terra nostra se le añade la expresión et aliunde venientes.

Con ello el convenio se abre a cuantos desearan participar y de hecho en la empresa intervienen catalanes, aragoneses, navarros bearneses, occitanos, italiarios, castellanos y franceses; y también no cristianos pues colectivos y particulares judíos recibieron tierras en el reparto.

\section{El operativo empresa marítimo terrestre}

3. El objetivo era el sometimiento del espacio balear: Insulas Majoricas, Minoricas, Eviçam et alias insulas que vocantur generaliter Baleares. Ello planteaba dificultades adicionales inéditas en buena parte. No se trata como en el caso de Lisboa, de Almería o de Tortosa de movilizar una flota en "función auxiliar", para que, desde el mar, bloqueando la llegada de socorros cooperara a una operación esencialmente terrestre. 
Era una operación inicialmente marítima en la que la flota tenía que asumir «funciones no auxiliares sino esenciales»: transportar pertrechos y efectivos combatientes, asegurar el reabastecimiento de la hueste durante la campaña y, sobre todo, garantizar el dominio del mar Baleat; dominio que, cual se comprobó en la frustada campaña catalano-pisana de 1114/1115 condicionaba sine qua non el éxito de la empresa.

En la Crónica Real se subraya la contribución de occitanos, sobre todo marselleses, para superar las dificultades estructurales, tácticas y logísticas planteadas no sólo en orden al transporte naval sino durante el asedio de la ciudad, en el que participan occitanos como expertos en técnicas artilleras, en montar artilugios de asalto a murallas y en la apertura de minas para abrir brechas en puntos adecuados, sobre experiencias de vanguardia adquiridas en operaciones de asedio a lugares fortificados de Tierra Santa que se aplicaron en el duro asedio de la ciudad de Mallorca.

\section{Táctica de guerra total}

4. En la reconquista hispánica las operaciones tácticas tendían a golpear al enemigo, desmantelando su moral combativa, para, en atención a prácticas consuetudinarias de cierta caballeresca civilidad, obligarle a negociar, a capitular; $y$ era corriente pactar treguas durante las operaciones de asedio. Las ciudades, por lo común, no se toman al asalto sino por capitulación.

En Mallorca la operación se desarrolló con modos distintos. En Mallorca se aplican tácticas de guerra total, que no otorgan cuartel al enemigo, desde una filosofía maximalista y prepotente de exterminio que pretende aniquilar al enemigo para, según prácticas más contemporáneas que medievales, imponerle la rendición incondicional.

Por ello el alto mando cristiano rechazó negociar una capitulación a todas luces ventajosa, y la ciudad tomada con fiereza al asalto fue sometida a un saqueo prolongado, sistemático y devastador. En Mallorca se aplicó con rigor el espíritu de cruzada.

5. ¿Por qué se aplica un táctica tan inmisericorde, casi inhabitual en el medievo hispánico? La Crónica Real argumenta «motivaciones de orden estratégico determinadas por circunstancias inherentes a la insula- 
ridad». Importaba tomar la ciudad pero sobre todo importaba asegurar la consolidación de la conquista, considerando la complejidad de movilizar y transportar efectivos con rapidez, en caso de necesidad, desde bases de tierra firme.

Dicha consolidación se entendía arriesgada de accederse -como pretendía el wali almohade Abu Yhaya- a facilitar ol transporte a Berbería de los musulmanes que pagando rescate (cinco besantes por cabeza) optaran por emigrar acaso con el propósito de promover desde Berbería un contragolpe.

También se consideraba arriesgada la permanencia en Mallorca de una masa importantes de musulmanes que, en el supuesto de contragolpe, pudieran actuar en su apoyo como quintacolumnistas. Desde tales convicciones de orden estratégico el «imperativo de afirmar la seguridad de lo conquistado" se sobrepuso a cualquier otra consideración de naturaleza económica o humanitaria.

\section{Integración de los musulmanes}

6. La operancia de presupuestos de orden estratégico explica otra peculiaridad. Tras la conquista sobreviven en Mallorca musulmanes libres y musulmanes cautivos cuya permanencia se considera conveniente y necesaria para promover la recuperación económica tras el duro trauma de la conquista; pero en ningún caso se les otorga, como era habitual que se les concediera en los reinos hispánicos, un Estatuto jurídico que les garantizara la conservación bajo la soberanía cristiana de su religión, de sus instituciones político-administrativas y de sus costumbres, y les reconociera como "comunidad autónoma de musulmanes de Mallorca» diferenciada de la «comunidad cristiana de Mallorca».

Dicho Estatuto fue otorgado en seguida a los judíos integrados en la denominada Universitatis Judeorum Calli Majoricarum, amparada en privilegios propios y con personalidad jurídica diferenciada de la comunidad cristiana o Universitatis civitatis et Regni Majoricarum. De esta manera la judería existío de inmediato pero no se institucionalizó la morería.

7. La Crónica Real menciona la sustanciación por escrito en 1231 de un convenio entre Jaime I y Xuaip, lider de los moros resistentes en la Cordillera Norte, que les facultaba por lo que se deduce, para perma- 
necer en Mallorca como hombres libres aunque desposeídos de su patrimonio.

En el tratado de protectorado de Capedepera concluido aquel año de 1231 entre Jaime । y los moros notables de Menorca se establece que los sarracenos menorquines que libremente se establecieran en' Mallorca gozarían del fuero de los sarracenos de Mallorca. ¿Qué fuero? No consta ningún fuero esencial. Lo que consta es que en Mallorca permanecían moros libres bajo el amparo y salvaguardia real, no integrados en morería, que pagan al rey por la protección una capitación anual.

Están documentados numerosos «baptizati» que en ciertos casos -por ejemplo los «Benasser», los «Benamar», los "Abenjohan" o los "Abenmartín»- conservan su linaje de la época musulmana aunque la identificación arrom que les aplica resulta desorientadora. ¿Eran conversos dichos arrom o eran cristianos de la Mallorca musulmana sólo islamizados en el modo de vida?... Lo normal es que a los «baptizati», a los conversos, se les designe con su nombre de pila y la identificación «baptizati» como, por ejemplo, «Petrus baptizati» 0 «Johannes baptizati».

Obra la necesaria información para pensar que el proceso de conversión tanto de los mulsumanes libres como de los musulmanes cautivos se realizó con cierta fluidez y, al parecer, sin presiones ambientales hostiles. Al efecto una resolución sinodal establece que podrá impartirse el bautismo sin precipitaciones a los que lo soliciten, sometiéndoles a un período de prueba, de reflexión, por lo menos de tres días.

8. Respecto a los mulsumanes de Mallorca que permanecieron como musulmanes "casatos" o como musulmanes cautivos están documentadas ya en el primer quinquenio de la postconquista fórmulas contractuales, como los "convenios de talla" o los "contratos laborales de redención de cautiverio», por cuyo conducto el cautivo al que el dueño le otorgara la gracia, a partir de la firma del contrato, que autoriza un notario y avala el batle real, goza de una situación de cuasi libertad en el sentido de que se le faculta para ejercer con autonomía su menester artesanal o para cultivar por su cuenta o en aparcería las tierras de labranza que se confían o para contratar sus servicios o terceras personas como menestrales o peones.

Tales cautivos agraciados por el contrato se administran con autonomía, circulan libremente por la Isla y pueden demandar al dueño ante el batle real si incumple las normas convenidas; y contraen la obligación 
de pagar puntualmente al dueño una cuota semanal, mensual o anual hasta el desvengo de la cantidad establecida en el contrato; por su parte, cumplimentado el contrato, el dueño se obliga a otorgarles instrumento de manumisión o "carta de alforría» que les acredita como moros libres, con libertad para permanecer en Mallorca pagando capitación o emigrar a tierras de moros o de cristianos.

9. Cumplida la conquista el tratamiento expeditivo, de notorio pragmatismo político, que se aplica en Mallorca tanto a los mulsumanes libres como a los mulsumanes cautivos propició su inserción, su integración como cristianos en los niveles inferiores de la nueva sociedad cristiana. Aunque los que conservaron su linaje, sobre todo los Bennasser («Johannnes Bannasser arrom»), asumen en seguida funciones en el área de los servicios como propietarios de tierras que sugieren su inserción en estratos sociales acomodados.

En conclusión, en el reino de Mallorca la política de bloquear la continuidad de los musulmanes como comunidad autónoma de musulmanes de Mallorca cumplió el objetivo de promover su cristianización y propició el que se insertaran en la nueva sociedad cristiana, aunque a comienzos del siglo xIV parece - según el testimonio del cronista Marsilio- que persistían musulmanes descendientes de musulmanes de la conquista.

En cuanto a los musulmanes foráneos, asentados como musulmanes libres o como moros cautivados por corsarios, instalados en Mallorca hasta la modernidad, pues la conquista no rompió las relaciones con la España musulmana y Bervería, no generaron problemáticas especiales.

\section{EL PROCESO REPOBLADOR}

10. El aserto de que «colonitzada e repoblada Mallorca pels catalans es va crear a l'illa un nou reialme ... unit a Catalunya", ha encontrado eco en la historiografía y ha generado estados de opinión muy potenciados en la actualidad. Tal aserto es un lapsus material irrelevante de Rovira i Virgili. 
Lapsus material irrelevante porque lo que explica a continuación Rovira i Virgili, historiador eminente y objetivo, es que «aquests nous territoris» - alude al reino de Mallorca y al reino de Valencia- «non van esser anexats a Catalunya ni a Aragó sino que van formar nous reialmes». Y saca a colación sin identificarlo el aserto de un vell historiador: "Cuanto el gran don Jaime el Consquistador se apoderó en Valencia arrojando a los moros no le dío forma de provincia, la hizo nación».

Rovira i Virgili hubiera podido sacar también a colación en apoyo de sus tesis sobre que ni Valencia ni Mallorca fueron unidos o anexionados ni a Cataluña ni a Aragón, la afirmación formulada a fines del siglo XVI por el protohistoriador de Mallorca don Juan Benimelis: "La isla de Mallorca és y ha sido reino por sí desde que fue tomada del señorío de los moros".

Con acertado criterio jurídico rechaza Rovira i Virgili la calificación, tan generalizada en la historiografía, que contempla la Corona de Aragón como Federación D Confederación. $Y$ explica: "No hi hagué un Estat compost, ans be Estats juxtaposats amb algunes relacions politiques creades per la comunitat de sobirà; per aixó nosaltres preferim al terme Unió de la Corona d'Aragó al de confederació de la Corona d'Aragó».

$\mathrm{Y}$, en efecto, la Corona de Aragón carente en el medievo de las instituciones de coordinación necesarias y requeridas para conformar lo que en Derecho Político Constitucional se entiende como Confederación, constituyó una Unión Personal enlazados los territorios que la integraban por la vinculación común a la soberanía de un monarca común.

En el último cuarto del siglo XIII se instrumenta un pacto conocido como "Pacto o Privilegio de la Unión", en el que el rey y representantes de los territorios que conformaban la Corona juramentan no romper la Unión ni separar territorios de la Unión. Unión que tiene denominación de origen constantemente documentada: «Corona de Aragón o Comunidad de la Corona de Aragón».

11. La Crónica de Ramón Muntaner escrita en 1325-1326 informa: «La illa de Mallorca es vuy... poblada toda de catalans tots d'honrats llocs e de bo». La Crónica Real más cerca a la realidad histórica manifiesta en la parte elaborada en torno a 1244 que en Valencia y en Mallorca permanecieron los sarracenos que se sometieron y que la tierra libre la poblaron cristianos. La Crónica de Bernat Desclot elaborada entre 1285 
y 1295 narra: «E així la ciutat de Mallorques se pobla de crestians... e tota l'illa».

Las fuentes prueban que la Crónica Real y la de Bernar Desclot están mejor informadas y son más objetivas que la de Ramón Muntaner, cronista aúlico, cuya prodigiosa capacidad para fabular, para presentar los héchos no como acaecieron sino como a su juicio tenían que haber acaecido, sólo es equiparable a su dominio de la lengua catalana y a su maestría literaria.

12. La proclividad a identificar la procedencia de los repobladores apoyándola sobre todo, como es práctica corriente en cierta historiografía actual, en las "áreas territoriales lingüísticas de apellidos", es decir, sobre el «actual diseño de la geografía de apellidos», es quehacer metodológico cómodo pero aventurado y de poco rigor dado que «el actual diseño es distinto al existente en los siglos XII y XIII».

Analizando las fuentes se comprueba que apellidos como Amat, Bord, Castell, Durán, Ferrer, Fontanes, Fornells, Más, Petit, Prat, Sabater, Vaquer, Vilanova o Vilella y un larguísimo etcétera, existían en los siglos XII y XIII -antes de la conquista de Baleares- en un amplia área geográfica que por lo menos va de Provenza a Navarra y de comarcas del Bajo Aragón, del sur del Ebro, a comarcas lemosinas y auvernienses del norte del Garona.

Tal fenómeno pienso que pudiera relacionarse con el «nivel de evolución lingüistica a partir del tronco común», la lengua latina, alcanzado por las modalidades lingüísticas romances todavía casi nacientes en los siglos XII y XIII, detectable en similitudes en nombres y en formas de expresión.

En la Crónica de Jaime I y en la de Bernat Desclot la locución nostre llati tenia el significado de lengua coloquial popular dimanante del latín todavía a un nivel de evolución lingüística que permitía que, por ejemplo, un provenzal de Marsella se entendiera sin ninguna dificultad con un aragonés de Calatayud o con un navarro de Pamplona o con un genovés o castellano o portugués o gallego.

13. Pienso que mientras no obren fuentes más idóneas cabe identificar la procedencia de los repobladores o aproximarse con más rigor metodológico a dicha identificación, «valiéndose de los locativos y genti- 
licios», es decir, de «topónimos utilizados como adjetivos en función de apellidos".

La cuantificación de los locativos y gentilicios empleados como apellidos de 136 enfiteutas que reciben entre 1232 y 1236. O sea en los años inmediatos a la conquista, casas en la ciudad de Mallorca, en la porción urbana del conde del Rosellón Nuño Sans, manifiesta que 54 de los enfiteutas $(39,71$ por 100$)$ procedían de Cataluña, $33(24,26$ por 100$)$ de Francia meridional, 27 (16,19 por 100) de Italia, 10 (7,35 por 100) de Aragón, 8 (5,88 por 100 de Navarra, 6 (4,42 por 100) de Francia central, $2(1,47$ por 100$)$ de Castilla y $1(0,73$ por 100$)$ de Flandes.

Tal cuantificación válida en cuanto al distrito urbano del conde Nuño Sans en los mentados años, pudiera sumirse «sólo con alcance provisional e indicativo" respecto al conjunto del proceso repoblador insular; confirmado con el criterio historiográfico de una repoblación de procedencia mayoritaria catalana $(39,71$ por 100 en el mentado distrito), pero no exclusiva ni hegemónicamente mayoritaria catalana. En Mallorca se asientan gentes muy diversas, pues la variedad de procedencias es mucho más plural que la que resulta de los expresados asentamientos urbanos.

14. Promovida por una legislación pensada para atraer repobladores a una tierra nueva de hombres libres la repoblación debió efectuar a ritmo satisfactorio.

«El rei partí la terra -explica la Crónica Real- e donala llir franca et quitia, sens dret e usatge - sin servidumbres-que no fossen tenguts de onar [los pobladors] a null home». Luego refiere al desarrollo económico alcanzado en torno a 1244-1252: «E de llahora ença - desde la conquista - Nostre senyor ha multiplicada l'illa de Mollorques que val - ara - dos tants que no faīa — que no valía - en lo temps dels sarrahins".

Las fuentes documentales confirman la afirmación de la Crónica Real respecto al desarrollo económico que de alguna manera se relaciona con el desarrollo demográfico ligado al proceso repoblador. Aportaré un dato significativo y revelador: "al cumplirse el primer centenario de la conquista en 1329 Mallorca" con una demografía de 12.454 fuegos "alcanza su apogeo demográfico medieval». En 1524, tras la revuelta de los agermanados la demografía era de 7.904 fuegos con una disminución respecto a 1329 del 30,20 por 100 . 
El mentado apogeo demográfico alcanzado al cumplirse el primer centario sugiere la "permanencia de una masa considerable de musulmanes convertidos" ya integrados plenamente en el nuevo tejido social; y el «asentamiento de repobladores cristianos a un ritmo acaso altamente satisfactorio", pues por las mismas calendas eran territorios en vías de repoblación comarcas situadas entre el Ebro y el Segura.

\section{SOCIEDAD PLURAL DE COMPONENTES BURGUESES}

15. La historiografía clásica catalana (Valls Taberner, Rovira i Virgili, Ferrrán Soldevila) y la historiografía mallorquina desde don Juan Benimelis a Mossèn Antoni Pons, incluidos entre otros don José María Quadrado, Benet Pons Fábregues y don Jaime Salvá, interpretan apoyándose en el análisis de las fuentes que «la conquista cristiana asienta en Mallorca un sistema social burgués», entendido por Rovira i Virgili como «règim democratic tant en l'aspecte social com el politic... que ha estat anomenat amb justicia Constitució del regne de Mallorca».

En la actualidad corrientes historiográficas desde posturas dialécticas muy significadas, de gran fiabilidad y audiencia en ambientes universitarios, no sólo ponen en cuarentena dicha interpretación clásica sino que la descalifican, a veces con marcado sarcasmo, como desfasada y obsoleta apoyada más en tópicos que en evidencias.

A tenor de tales corrientes historiográficas desmitificadoras y renovadoras «lo que asienta en Mallorca la conquista cristiana es una sociedad feudal», que cierta historiografía ponderada y proclive al posibilismo describe como «sociedad feudal de connotaciones peculiares» que tipifican el denominado «feudalismo mallorquín».

Dada la importancia y actualidad de la temática y la gran implantación que ha alcanzado la versión de Mallorca como sociedad feudal, pienso que hace el caso asentar ciertas precisiones con ánimo de contribuir a clarificar el tema desde la objetividad, situándolo en el área que le corresponde.

16. Lo que la conquista que las crónicas del siglo XIII definen como "conquista de la cristiandad", asienta en Mallorca es "una sociedad plu- 
ral» en la que coexisten con «comportamientos sociales mayoritarios» afines a lo que Sombart denomina "espíritu burgués» propio de lo que entiende como "primer capitalismo", y a lo que Le Goff considera "burguesía mercantil», con "comportamientos minoritarios feudales" que no autorizan a definirla, a no ser que medie desinformación o voluntarismo, dado que las sociedades, normalmente plurales, se definen por sus características más esenciales y en el caso del reino de Mallorca-salvando ciertas peculiaridades ibicencas-dichas características son similares a las constatadas desde los siglos XII y XIII en centros mercantiles de Italia, de Occitanis, de Francia del Norte y de Flances.

17. En las áreas rurales del reino de Mallorca importa considerar por lo menos los siguientes factores:

a) «El prodominio hegemónico de la propiedad alodial» no sometida - como relata la Crónica Real y prueban los contratos de concesión de tierras- a servidumbres señoriales; predominio en relación a las tendencias feudales centradas en las denominadas «caballerías», es decir, tierras o rentas en dinero sometidas a la obligación de mantener uno o más caballos armados en defensa del reino de Mallorca, como cuerpo montado comandado por el gobernador como alter ego del rey, en función de reserva de intervención inmediata en caso de necesidad. Eran en Mallorca como un centenar de caballos forzados.

b) «El predominio hegemónico de estructuras agrarias de superficie pequeña y mediana" (entre 10 hectáreas y 60 hectáreas), con unidades agrarias familiares que solían tener dos jouades - de jou, yugo- o yugadas equivalentes a una superficie de 22 hectáreas laborable con una yunta de bueyes (Duas jouatas sarracenicas -especifica un contrato de asentamiento enfitéutico de 1242 — sint suficientes ad laborandum unius par bovis).

En Mallorca se consideraba que una suerte de dos yugadas de tierra era unidad de cultivo adecuado a la labranza anual de una yunta de bueyes y, por lo que se desprende, al mantenimiento de una explotación familiar campesina y al pago del censo alodial y del censo enfitéutico que en su caso correspondiera.

c) «La tenencia de las tierras por campesinos libres» sin que esté documentada la existencia de siervos vinculados a la tierra aunque sí la de numerosos esclavos al servicio de los propietarios alodiales, de los tenedores de «caballerías» y de los poseedores enfitéuticos del dominio 
útil de las tierras. Tales campesinos que poseen tierras en enfiteusis predominaban hegemónicamente sobre los campesinos aparceros y los campesinos arrendatarios.

La enfiteusis, que permite acceder a la cuasi propiedad de la tierra y otorga sobre la misma no un derecho personal - como en el caso de la aparcería o el arrendamiento- sino un derecho real sobre la tierra, sin pagar precio en concepto de compra de la tierra, con libre disposición para transmitirla hereditariamente o para transferirla a terceros mediando -en este supuesto- la «fatiga» y el debengo al señor del dominio directo de la correspondiente «cuota laudemial» (entre el 6 y el 30 por 100 del precio de la transferencia), se implanta en Mallorca en la inmediata postconquista y conserva vigencia - actualizada por normas del «Parlament Balear»- en la actualidad.

Y cuando una institución se mantiene operante durante tantos siglos es porque a escala humana es socialmente justa y, en lo posible, económicamente adecuada y eficaz.

d) «La existencia en Mallorca de villas rurales" - exactamente 33 municipios- "sometidas al batle real», que asume la justicia como delegado del rey, y administradas por Consejos Municipales renovados cada año, estructurados a veces en manos (mano mayor, mano mediana, mano menor), presididos por el «batle real o integrados por «jurats» -en general 4 jurats - con funciones ejecutivas, y cierto número ordenan los pagos comunitarios que cumplimenta el "clavari», que maneja los dineros municipales.

«Jurats» y «claveri», finalizado el mandato anual, responden de su gestión ante "oydors de comptes» o "comptadors", en general tres contadores elegidos por el Consejo Municipal y renovados cada año, que prestan juramente ante el batle real y auditan la contabilidad municipal y pronuncian - comprobadas las partidas de «rebudes»o ingresos y de "dades" o gastos y el "por qué» y el "para qué" del gasto realizadola sentencia que correspondiera, de caracter ejecutivo.

e) "La agrupación de las villas rurales" desde la primera mitad del siglo XIV «en el denominado Sindicat de les Viles Foranes de Mallorques», asamblea político-administrativa de naturaleza representativa y renovación anual, gobernada por un Consejo Ejecutivo de diez miembros - los "sindics forans" - y compuesta por "consellers" representantes de los municipios foráneos en el número que, según la contribución fiscal al 
gasto del Sindicato, a más contribución más consellers, correspondiera a la villa.

El "Sindicat de les Viles Foranes" coordina y defiende los intereses del colectivo de las comarcas rurales frente a la prepotencia de la oligarquía burguesa instalada en el núcleo urbano de Mallorca. Dicha defensa normalmente se ejercita con buen sentido y serenidad, ánimo dialogante y negociador y frecuente apelación a la función arbitral de la Corona bien mediando su «alter ego», el gobernador, bien apelando directamente a la Corona para que, en su caso, reponga los intereses o derechos conculcados.

«Así como nuestro cuerpo, sin mandato de la mente, no puede usar de sus miembros" - le decía en 1424 Franscesh del Postigo abogado asesor del "Sindicat" al gobernador de Mallorca Olfo de Próxida-, "asi mismo un reino no puede vivir si no se observan las leyes". Y tras la frustración de la tremenda revuelta foránea de 1450 o 1452, los emisarios foráneos le recordaron al rey Alfonso el Magnánimo en Nápoles en 1454: «El reino de Mallorca desde sus orígenes ha sido fundamentado de libertad y manantial de igualdad».

Sentimientos de libertad e igualdad que ordinariamente inspiraron los comportamientos del "Sindicat" en el bajo medievo y en tránsito a la modernidad e incluso en la modernidad, aunque con mucho timidez, pues la regresión social descabalgada la burguesía, en conjunción con los cambios económicos, habían señorializado la administración en una Mallorca sometida a los desafueros de las banderías señoriales y de un bandidismo que operaba con impunidad.

Pero en el medievo era distinto. La cohesión del campesinado foráneo es el motor que dinamiza el «pogrom" de 1391, con la devastación de la judería de Inca y de la poderosa judería de la ciudad, cuando los judíos de Mallorca ejercieron como cabeza de turco de planteamientos sociales más profundos; pues la revuelta, iniciada como antisemítica derivó contra la oligarquía burguesa y se materializó en el saqueo de algunas casas de significados burgueses.

La fuerza del «Sindicat de les Viles Foranes» se manifestó en la revuelta de 1450-1452 cuando el campesinado impuso su ley en Mallorca hasta que comandos de tropas mercenarias profesionales enviadas por la Corona desde Nápoles sometieron al campesinado. Dicha revuelta por su larga duración, por la voluntad política, capacidad de organización y 
potencia de recursos es el movimiento agrario más esencial y significado del Occidente europeo en el bajo medievo.

La fuerza del Sindicat de les Viles Foranes volvió a manifestarse en 1520 pues la revuelta de los agermanados se consolida en Mallorca cuando los activistas foráneos se alinean con los activistas menestrales urbanos iniciadores de la revuelta.

18. En el área urbana de Mallorca el modelo burgués de sociedad asentado en la postconquista, en la inmediata postconquista, se denota:

a) "Por el grado de implantación de la menestralia" patente en la rama de "curtidos y manufacturas de pieles" que desde la primera década de la postconquista cuenta con una estructura competitiva que le permite exportar; y en la «rama textil» en la que ante el obsoleto montaje textil heredado - como la curtidaría - de la época islámica, se plantea la necesidad de renover tácnicas para competir por lo menos en el mercado local insular; y ello acaece tempranamente pues obra, según mis fuentes, un proyecto de 1247 para fabricar en Mallorca ad consuetudinem Narbone, según técnica narbonesa, paños de tres calidades: de estambre plano, de estambres listado y de lana Bugía.

b) «Por el temprano y potente desarrollo de armamentos corsarios» organizados desde 1230 - año de la conquista - como "actividad normalizada», como negocio remunerador, acaso el más remunerador, en operaciones financieras sobre todo por gentes modestas que aportan su escaso peculio o sus personas para armar pequeñas barcas ad lucrum contra sarracenos. Estas pequeñas empresas que operan en seguida ad consuetudinem Maiorice, o costumbre marítima de Mallorca, pienso que ejercieron como fuente principal de formación de capitales en la inmediata postconquista. Capitales que alimentan el tráfico mercantil y la recuperación agraria.

c) «El desarrollo del tráfico mercantil» favorecido por la función del área balear como «plataforma de depósito y redistribución de mercancías", activada sobre todo por la colonia genovesa y en menor medida por mercaderes occitanos asentados en Mallorca promotores de fletes causa negociandi.

Desde el área balear arrancan rutas que discurren por todos los cuadrantes de la rosa marinera con especial incidencia en la ruta Sur hacia Berbería y en la ruta del Sudoeste hacia la España musulmana, 
que a una década de la conquista, en el trienio 1240-1243, canalizan el 41,86 por 100 de la navegación mercantil que tengo documentada.

Dicho elevado coeficiente no es extraño pues la conquista cristiana no rompe las relaciones mercantiles con el área islámica existentes en la época musulmana, sino que contribuye a potenciarlas capitalizando la circunstancia, notoria desde los albores de la navegación mediterránea, del área balear como charnela del tráfico mediterráneo.

La mercadería, como reiteran tenazmente las fuentes, es el eje y el soporte de las estructuras del reino de Mallorca hasta la Modernidad. Y también de las estructuras políticas pues son los mercaderes y los ciudadanos que ejercen como mercaderes los que dominan hasta el siglo xv en el Consell General.

d) «Por el hecho de que desde la primera década de la post-conquista» la economía del reino de Mallorca funciona como «arquetipo de economía dineraria de cuño burgués, en las antípodas de la economía monetaria feudal. Por ejemplo, sobre un total analizado de 360 contratos enfitéuticos urbanos de la primera década tras la conquista -número cuantitativamente significativo- sólo en cinco casos el censo se contrata en especie; en el resto se conviene censo pagadero en dineros, lo que objetiva un coeficiente del 98,65 por 100 de censos en dinero frente a 1,35 por 100 de censos en especie.

e) «Porque tras la conquista se establece de inmediato un sistema monetario abierto». Abierto a cualquier moneda circulante, aunque la cuantificación monetaria practicada evidencia que en el primer trienio (1230-1233) la moneda que en régimen abierto circular mayoritariamente es la macemutina jucefia, pudiera ser que en línea de continuidad con la situación monetaria existente en la breve fase - como una treintena de años- de dominio almohade en Mallorca.

Circulación mayoritaria porque más del 60 por 100 de la negociación y contratación documentada se contabiliza en macemutinas jucefias; pero desde 1233 la divisa que circula en mercado abierto mayoritariamente es la moneda «melgaresa», propia de Montpeller cuyos cónsules coasociados con el obispo de Melgeuil y con el rey de Aragón como señor de Montpeller cobran los derechos establecidos sobre la acuñaciones de «melgareses». 
Circulación hegemónica del «melgarés» porque sobre un total de 570 referencias monetarias - que no son pocas referencias- documentadas en el año 1242, sólo en dicho año, a sólo doce años de la conquista, el 81,81 por 100 son "melgareses", el 7,94 por 100 «besantes", el 4,84 por 100 «morabatines alfonsinos», el 3,11 por 100 «barceloneses" y el 1 por 100 «jaqueses».

El sistema monetario abierto se cierra en 1247 cuando, probablemente a instancias de emisarios del reino de Mallorca en respuesta a sugerencias del rey, se declara como moneda legal de circulación obligada y contractualmente exclusiva del reino de Mallorca el «real de Valencia" creado aquel mismo año 1247. Luego, en 1300, Jaime II de Mallorca creará el "sistema monetario propiamente mallorquín" cuya vigencia se mantiene con diversos reajustes -en especial la fundamental "reforma monetaria de Fernando el Católico» de 1508- hasta el siglo XVIII. 